Portland State University

PDXScholar

Mathematics and Statistics Faculty

Fariborz Maseeh Department of Mathematics

Publications and Presentations

and Statistics

$10-1-2000$

Dependence Among Spacings

Baha-Eldin Khaledi

Subhash C. Kochar

Portland State University

Follow this and additional works at: https://pdxscholar.library.pdx.edu/mth_fac

Part of the Mathematics Commons

Let us know how access to this document benefits you.

Citation Details

Khaledi, E. and Kochar, S. (2000). Dependence among spacings. Probability in the Engineering and Informational Sciences, 14, pp 461-472.

This Article is brought to you for free and open access. It has been accepted for inclusion in Mathematics and Statistics Faculty Publications and Presentations by an authorized administrator of PDXScholar. Please contact us if we can make this document more accessible: pdxscholar@pdx.edu. 


\title{
DEPENDENCE AMONG SPACINGS
}

\author{
Baha-Eldin Khaledi and Subhash Kochar \\ Indian Statistical Institute \\ New Delhi-110016, India \\ E-mail:kochar@isid.ac.in
}

\begin{abstract}
In this paper, we study the dependence properties of spacings. It is proved that if $X_{1}, \ldots, X_{n}$ are exchangeable random variables which are $\mathrm{TP}_{2}$ in pairs and their joint density is log-convex in each argument, then the spacings are $\mathrm{MTP}_{2}$ dependent. Next, we consider the case of independent but nonhomogeneous exponential random variables. It is shown that in this case, in general, the spacings are not $\mathrm{MTP}_{2}$ dependent. However, in the case of a single outlier when all except one parameters are equal, the spacings are shown to be $\mathrm{MTP}_{2}$ dependent and, hence, they are associated. A consequence of this result is that in this case, the variances of the order statistics are increasing. It is also proved that in the case of the multiple-outliers model, all consecutive pairs of spacings are $\mathrm{TP}_{2}$ dependent.
\end{abstract}

\section{INTRODUCTION}

Let $X_{1}, \ldots, X_{n}$ be $n$ random variables. We shall denote by $X_{i: n}$ the $i$ th-order statistic of $X_{1}, \ldots, X_{n}$. Let $D_{i: n}=X_{i: n}-X_{i-1: n}$ denote the $i$ th spacing, $i=1, \ldots, n$, with $X_{0: n} \equiv 0$. It is well known that if $X_{1}, \ldots, X_{n}$ is a random sample from an exponential distribution, then $D_{1: n}, \ldots, D_{n: n}$ are independent. In this paper, we study the dependence properties of spacings when $X_{i}$ 's are not necessarily independent and identically distributed as exponentials. The related problem of stochastic orderings among spacings has been extensively studied in the literature. For details, the reader is referred to a recent review paper on this topic by Kochar [9]. Throughout this paper, increasing means nondecreasing and decreasing means nonincreasing.

There are several notions of positive and negative dependences among random variables with varying degrees of strength. There is a vast literature on this topic, with important contributions by Lehmann [11], Esary and Proschan [3], Barlow and Proschan [1], Block and Ting [2], and Karlin and Rinott [6,7], among others. Per- 
haps the strongest notion of positive dependence between random variables $S$ and $T$ is that of $\mathrm{TP}_{2}$ dependence (also known as likelihood dependence). $S$ and $T$ are $\mathrm{TP}_{2}$ dependent if their joint density $f(s, t)$ is totally positive of order 2 in $s$ and $t$, or, more precisely, if

$$
\left|\begin{array}{ll}
f\left(s_{1}, t_{1}\right) & f\left(s_{1}, t_{2}\right) \\
f\left(s_{2}, t_{1}\right) & f\left(s_{2}, t_{2}\right)
\end{array}\right| \geq 0
$$

whenever $s_{1}<s_{2}$ and $t_{1}<t_{2}$.

We say that $T$ is right-tail increasing in $S$ if $P[T>t \mid S>s]$ is increasing in $s$ for all $t$, and we denote this relationship by $\operatorname{RTI}(T \mid S)$. Finally, random variables $S$ and $T$ are associated [written $A(S, T)]$ if $\operatorname{cov}[\Gamma(S, T), \Delta(S, T)] \geq 0$ for all pairs of increasing binary functions $\Gamma$ and $\Delta$. As shown in Barlow and Proschan [1, p. 143], the following chain of implications holds among the above notions of positive dependence:

$$
\mathrm{TP}_{2}(S, T) \Rightarrow \operatorname{RTI}(S \mid T) \Rightarrow A(S, T) .
$$

There are many other notions of dependence, but we will not discuss them here.

These concepts of bivariate dependence can be easily extended to the multivariate case. A function $\psi: R^{n} \rightarrow[0, \infty)$ is said to be multivariate total positivity of order 2 (denoted by $\mathrm{MTP}_{2}$ ) if

$$
\psi(\mathbf{x}) \psi(\mathbf{y}) \leq \psi(\mathbf{x} \wedge \mathbf{y}) \psi(\mathbf{x} \vee \mathbf{y}) \quad \text { for every } \mathbf{x} \text { and } \mathbf{y} \text { in } \mathcal{R}^{n},
$$

where $\mathbf{x} \wedge \mathbf{y}=\left(\min \left(x_{1}, y_{1}\right), \ldots, \min \left(x_{n}, y_{n}\right)\right)$ and $\mathbf{x} \vee \mathbf{y}=\left(\max \left(x_{1}, y_{1}\right)\right.$, $\left.\ldots, \max \left(x_{n}, y_{n}\right)\right)$. Random variables $X_{1}, \ldots, X_{n}$ are said to be $\mathrm{MTP}_{2}$ dependent if their joint density function is $\mathrm{MTP}_{2}$. It is shown in Kemperman [8] (see also Block and Ting [2]) that if the support of a random vector $\mathbf{X}=\left(X_{1}, \ldots, X_{n}\right)$ is a lattice (i.e., if $\mathbf{x}$ and $\mathbf{y}$ are in the support of $\mathbf{X}$, then so are $\mathbf{x} \wedge \mathbf{y}$ and $\mathbf{x} \vee \mathbf{y}$ ), then $X$ is $\mathrm{MTP}_{2}$ if and only if its density function $f$ is $\mathrm{TP}_{2}$ in each pair of its variables when the other $n-2$ variables are held fixed. See Karlin and Rinott [6] for more details on the properties of $\mathrm{MTP}_{2}$ functions. Random variables $X_{1}, \ldots, X_{n}$ are conditionally increasing in sequence if $P\left[X_{i}>x \mid X_{1}=x_{1}, \ldots, X_{i-1}=x_{i-1}\right]$ is increasing in $x_{1}, \ldots, x_{i-1}$ for $i=2, \ldots, n$. Finally, a set of random variables $\mathbf{X}=\left(X_{1}, \ldots, X_{n}\right)$ are associated if $\operatorname{cov}(u(\mathbf{X}), v(\mathbf{X})) \geq 0$ for all increasing binary functions $u$ and $v$. Karlin and Rinott [6] proved that if a set of random variables are $\mathrm{MTP}_{2}$ dependent, then they are conditionally increasing in sequence, which, in turn, implies that they are associated (cf. Barlow and Proschan [1, p. 146]), a result which extends (1.2) to the multivariate case.

It is known that spacings of a random sample from a DFR (decreasing failure rate) distribution are conditionally increasing in sequence (cf. Barlow and Proschan [1, p. 151]). Karlin and Rinott [6] have pointed out that if the DFR assumption is strengthened to assume that the parent distribution has a log-convex density, then the spacings have the corresponding stronger property of being $\mathrm{MTP}_{2}$ dependent. In Section 2, we extend this result to the case when the random variables $X_{1}, \ldots, X_{n}$ are 
dependent. It is proved that if the joint probability density function (p.d.f.) of $X_{i}$ 's is permutation symmetric, $\mathrm{TP}_{2}$ in pairs, and log-convex in each argument, then their spacings are $\mathrm{MTP}_{2}$ dependent (Theorem 2.1). In Section 3, we study the dependence properties of spacings of independent but nonidentically distributed exponential random variables. We show with the help of a counterexample that in this case, the spacings may not be $\mathrm{MTP}_{2}$ dependent. In fact, for $n=3$, even $\operatorname{RTI}\left(D_{3: 3} \mid D_{2: 3}\right)$ does not hold for some values of the parameters (Example 3.1). However, it is shown that $\operatorname{cov}\left(D_{2: 3}, D_{3: 3}\right)$ is nonnegative (Corollary 3.1) due to its Schur convexity (Theorem 3.2). It is also proved that in the case of a single outlier when all except one of the parameters are equal, the spacings are $\mathrm{MTP}_{2}$ dependent (Theorem 3.4). A consequence (Corollary 3.2) of this result is that in this case, $\operatorname{var}\left(X_{1: n}\right) \leq$ $\operatorname{var}\left(X_{2: n}\right) \leq \cdots \leq \operatorname{var}\left(X_{n: n}\right)$. We also prove that in the case of the multiple-outliers model (Theorem 3.5), any pair of consecutive spacings $D_{i: n}$ and $D_{i+1: n}$ are $\mathrm{TP}_{2}$ dependent for $i=1, \ldots, n-1$.

\section{DEPENDENCE AMONG SPACINGS OF EXCHANGEABLE RANDOM VARIABLES}

As pointed out in Karlin and Rinott [6, p. 483], the spacings of a random sample from a distribution with log-convex density are $\mathrm{MTP}_{2}$ dependent. In Theorem 2.1, we extend this result to the case when random variables are exchangeable and $\mathrm{TP}_{2}$ in pairs.

THeOrem 2.1: Let $X_{1}, \ldots, X_{n}$ be exchangeable random variables with absolutely continuous joint p.d.f. $f_{\mathbf{X}}\left(x_{1}, \ldots, x_{n}\right)$, which is positive on $\Pi_{i=1}^{n} \Omega_{i}^{n}, \Omega_{i} \subset \mathcal{R}^{1}$, $i=1, \ldots, n$, and satisfies the following conditions:

(a) $f_{\mathbf{X}}$ is $T P_{2}$ in pairs.

(b) $f_{\mathbf{X}}$ is log-convex in each argument when remaining arguments are held fixed.

(c) The first partial derivative of $f_{\mathbf{X}}(\mathbf{x})$ with respect to $x_{i}$ exists for $i=1, \ldots, n$. Then, $D_{1: n}, \ldots, D_{n: n}$ are $M T P_{2}$ dependent.

Proof: The joint p.d.f. of $D_{1: n}, \ldots, D_{n: n}$ is

$$
f_{\mathbf{D}}\left(d_{1}, \ldots, d_{n}\right)=n ! f_{\mathbf{x}}\left(d_{1}, \sum_{j=1}^{2} d_{j}, \ldots, \sum_{j=1}^{i} d_{j}, \ldots, \sum_{j=1}^{n} d_{i}\right) .
$$

By Theorem 1.5 of Karlin [5], $f_{\mathbf{D}}\left(d_{1}, \ldots, d_{n}\right)$ will be $\mathrm{TP}_{2}$ in pairs of $d_{1}, \ldots, d_{n}$ if and only if for any $i \neq j, 1 \leq i, j \leq n,\left(\partial / \partial d_{i}\right) \log f_{\mathbf{D}}\left(d_{1}, \ldots, d_{n}\right)$ is increasing in $d_{j}$. Let $i<j$. By the chain rule of differentiation,

$$
\left(\frac{\partial}{\partial d_{i}}\right) \log f_{\mathbf{D}}\left(d_{1}, \ldots, d_{n}\right)=\sum_{k=i}^{n}\left(\frac{\partial}{\partial x_{k}}\right) \log f_{\mathbf{X}}\left(d_{1}, \sum_{j=1}^{2} d_{j}, \ldots, \sum_{j=1}^{i} d_{j}, \ldots, \sum_{j=1}^{n} d_{i}\right),
$$


where $x_{k}=\sum_{l=1}^{k} d_{l}$ for $k \in\{1, \ldots, n\}$. The term $\left(\partial / \partial x_{k}\right) \log f_{\mathbf{X}}(\mathbf{x})$ is increasing in $x_{k}$ for $k \in\{1, \ldots, n\}$, as $f_{\mathbf{X}}$ is log-convex in $x_{k}$ for each $k$. It is increasing in $x_{m}, m \neq k$, $m \in\{1, \ldots, n\}$ because $f_{\mathbf{X}}$ is $\mathrm{TP}_{2}$ in pairs. Now, $x_{m}$ and $x_{k}$ are both increasing functions of $d_{j}$. This implies that $\left(\partial / \partial d_{i}\right) \log f_{\mathbf{D}}\left(d_{1}, \ldots, d_{n}\right)$ is an increasing function of $d_{j}$. Hence, $f_{\mathbf{D}}\left(d_{1}, \ldots, d_{n}\right)$ is $\mathrm{TP}_{2}$ in pairs. Clearly, the support of spacings is a lattice under the given conditions. Combining these facts, we get the required result.

Remark: In Theorem 2.1, if instead of conditions (a) and (b), we assume that $f_{\mathbf{X}}$ is $\mathrm{RR}_{2}$ (reverse regular of order 2) [two random variables $S$ and $T$ are $\mathrm{RR}_{2}$ dependent if the inequality in (1.1) is reversed] in pairs and $f_{\mathbf{X}}$ is $\log$-concave in each argument, then one can prove that the joint p.d.f. of spacings is $R_{2}$ in pairs.

Lemma 2.1: For a bivariate random vector $(X, Y)$,

$$
\operatorname{cov}(Y-X, X) \geq 0 \Rightarrow \operatorname{var}(X) \leq \operatorname{var}(Y) .
$$

Proof: The inequality $\operatorname{cov}(Y-X, X) \geq 0$ implies $\operatorname{cov}(X, Y) \geq \operatorname{var}(X)$, which, in turn, implies that

$$
\left\{\frac{\operatorname{var}(X)}{\operatorname{var}(Y)}\right\} \leq \rho^{2}(X, Y) \leq 1,
$$

where $\rho(X, Y)$ is the correlation coefficient between $X$ and $Y$. The required result follows from this.

This lemma and Theorem 2.1 lead to the following interesting corollary.

Corollary 2.1: Under the assumptions of Theorem 2.1,

$$
\operatorname{var}\left(X_{1: n}\right) \leq \operatorname{var}\left(X_{2: n}\right) \leq \cdots \leq \operatorname{var}\left(X_{n: n}\right) .
$$

Proof: Because under the given conditions $D_{i: n}$ 's are $\mathrm{MTP}_{2}$ dependent, they are associated. This implies that for $n=1, \ldots, j-1$,

$$
\operatorname{cov}\left(X_{j: n}-X_{j-1: n}, X_{j-1: n}\right) \equiv \operatorname{cov}\left(D_{j: n}, \sum_{i=1}^{j-1} D_{i: n}\right) \geq 0,
$$

as $\sum_{i=1}^{j-1} D_{i: n}$ and $D_{j: n}$ are increasing functions of $\left(D_{1: n}, \ldots, D_{n: n}\right)$. The required result follows from Lemma 2.1.

Example 2.1 (Inverted Dirichlet Distribution): Let $X_{i}, i=0, \ldots, n$, be independent gamma random variables each with scale parameter 1 such that $X_{0}$ has shape parameter $\beta$ and $X_{i}$ has shape parameter $\alpha$, for $i \in\{1, \ldots, n\}$. Then, the joint p.d.f. of $Y_{i}=X_{i} / X_{0}, i=1, \ldots, n$, is

$$
f_{Y_{1}, \ldots, Y_{n}}\left(y_{1}, \ldots, y_{n}\right)=\frac{\Gamma(n \alpha+\beta)}{(\Gamma(\alpha))^{n} \Gamma(\beta)} \frac{\left(\prod_{i=1}^{n} y_{i}\right)^{\alpha-1}}{\left(1+\sum_{i=1}^{n} y_{i}\right)^{n \alpha+\beta}} \quad \text { for } y_{i} \geq 0 .
$$


It is easy to see that $f_{Y_{1}, \ldots, Y_{n}}\left(y_{1}, \ldots, y_{n}\right)$ is exchangeable, $\mathrm{TP}_{2}$ in pairs, and log-convex in each argument when $0<\alpha<1$ and $n \alpha+\beta \geq 1$. Thus, the conditions of Theorem 2.1 are satisfied, and as a result, the spacings of $Y_{1}, \ldots, Y_{n}$ are $\mathrm{MTP}_{2}$ dependent. By Corollary 2.1, the variances of the successive order statistics increase as $i$ goes from 1 to $n$.

\section{THE CASE OF HETEROGENEOUS EXPONENTIALS}

The exponential distribution plays a central role in reliability theory. In this section, we study the dependence properties of spacings when the observations $X_{1}, \ldots, X_{n}$ are independent with $X_{i}$ having exponential distribution with parameter $\lambda_{i}, i=1, \ldots, n$. Their joint density is given by (cf. Kochar and Korwar [10])

$$
f_{D_{1: n}, \ldots, D_{n: n}}\left(x_{1}, \ldots, x_{n}\right)=\sum_{(\mathbf{r})} \frac{\prod_{i=1}^{n} \lambda_{i}}{\prod_{i=1}^{n} \sum_{j=i}^{n} \lambda\left(r_{j}\right)} \prod_{i=1}^{n}\left(\sum_{j=i}^{n} \lambda\left(r_{j}\right)\right) \exp \left\{-x_{i} \sum_{j=i}^{n} \lambda\left(r_{j}\right)\right\}
$$

for $x_{i} \geq 0, i=1, \ldots, n$, where $(\mathbf{r})=\left(r_{1}, \ldots, r_{n}\right)$ is a permutation of $(1, \ldots, n)$ and $\lambda(i)=\lambda_{i}$. It is a mixture of products of exponential distributions. From (3.1), it is easy to find that the joint p.d.f. of $\left(D_{i: n}, D_{j: n}\right)$ for $1 \leq i<j \leq n$ is

$$
\begin{aligned}
f_{D_{i: n}, D_{j: n}}(x, y)= & \sum_{(\mathbf{r})} \frac{\prod_{i=1}^{n} \lambda_{i}}{\prod_{i=1}^{n} \sum_{j=i}^{n} \lambda\left(r_{j}\right)} \\
& \times\left(\sum_{m=i}^{n} \lambda\left(r_{m}\right)\right) \exp \left\{-x \sum_{m=i}^{n} \lambda\left(r_{m}\right)\right\}\left(\sum_{m=j}^{n} \lambda\left(r_{m}\right)\right) \exp \left\{-y \sum_{m=j}^{n} \lambda\left(r_{m}\right)\right\}
\end{aligned}
$$

for $x, y \geq 0$.

The next example shows that the spacings may not be $\mathrm{MTP}_{2}$ dependent if the $\lambda_{i}$ 's are all different.

Example 3.1: Let $X_{1}, X_{2}$, and $X_{3}$ be independent exponential random variables with respective hazard rates 5,4 , and 1 . Using (3.2), we find, after some simplifications, that

$$
\begin{aligned}
h(y) & =P\left(D_{3: 3}>2 \mid D_{2: 3}>y\right) \\
& =\frac{20 e^{20 y}\left(\left(\frac{1}{9 e^{9 y}}+\frac{1}{6 e^{6 y}}\right)\left(\frac{1}{5}\right) e^{-10}+\left(\frac{1}{9 e^{9 y}}+\frac{1}{5 e^{5 y}}\right)\left(\frac{1}{4}\right) e^{-8}+\left(\frac{1}{6 e^{6 y}}+\frac{1}{5 e^{5 y}}\right) e^{-2}\right)}{e^{11 y}+4 e^{14 y}+5 e^{15 y}} .
\end{aligned}
$$


It is clear from Figure 1 that the function $h(y)$ is not monotonically increasing, proving thereby that $D_{3: 3}$ is even not RTI in $D_{2: 3}$. Hence, $D_{2: 3}$ and $D_{3: 3}$ are not $\mathrm{TP}_{2}$ dependent.

The covariance between $D_{i: n}$ and $D_{j: n}$ for $i<j$ is

$$
\begin{aligned}
\operatorname{cov}\left(D_{i: n}, D_{j: n}\right)= & \sum_{(\mathbf{r})} \frac{\prod_{i=1}^{n} \lambda_{i}}{\prod_{i=1}^{n} \sum_{j=i}^{n} \lambda\left(r_{j}\right)}\left\{\sum_{m=i}^{n} \lambda\left(r_{m}\right)\right\}^{-1}\left\{\sum_{m=j}^{n} \lambda\left(r_{m}\right)\right\}^{-1} \\
& -\left[\sum_{(\mathbf{r})} \frac{\prod_{i=1}^{n} \lambda_{i}}{\prod_{i=1}^{n} \sum_{j=i}^{n} \lambda\left(r_{j}\right)}\left\{\sum_{m=i}^{n} \lambda\left(r_{m}\right)\right\}^{-1}\right]\left[\sum_{(\mathbf{r})} \frac{\prod_{i=1}^{n} \lambda_{i}}{\prod_{i=1}^{n} \sum_{j=i}^{n} \lambda\left(r_{j}\right)}\left\{\sum_{m=j}^{n} \lambda\left(r_{m}\right)\right\}^{-1}\right] .
\end{aligned}
$$

We conjecture that, in general, the covariance between $D_{i: n}$ and $D_{j: n}$ for $i<j$ is nonnegative. We prove this conjecture for $n=3$ in Corollary 3.1. In fact, we prove in Theorem 3.2 that the covariance between $D_{2: 3}$ and $D_{3: 3}$ is Schur convex in $\lambda_{i}$ 's.

Let $\left\{x_{(1)} \leq x_{(2)} \leq \cdots \leq x_{(n)}\right\}$ denote the increasing arrangement of the components of the vector $\mathbf{x}=\left(x_{1}, x_{2}, \ldots, x_{n}\right)$. The vector $\mathbf{y}$ is said to majorize the vector $\mathbf{x}$ (written $\mathbf{x} \stackrel{m}{\sim} \mathbf{y}$ ) if $\sum_{i=1}^{j} y_{(i)} \leq \sum_{i=1}^{j} x_{(i)}$ for $j=1, \ldots, n-1$ and $\sum_{i=1}^{n} y_{(i)}=\sum_{i=1}^{n} x_{(i)}$. Functions that preserve the ordering of majorization are said to be Schur convex; that is, a real function $\phi$ defined on a set $\mathcal{A} \subset R^{n}$ is said to be Schur convex on $\mathcal{A}$ if $\mathbf{x} \stackrel{m}{\sim} \mathbf{y} \Rightarrow \phi(\mathbf{x}) \leq \phi(\mathbf{y})$. See Marshall and Olkin [12, Chap. 3] for properties and more

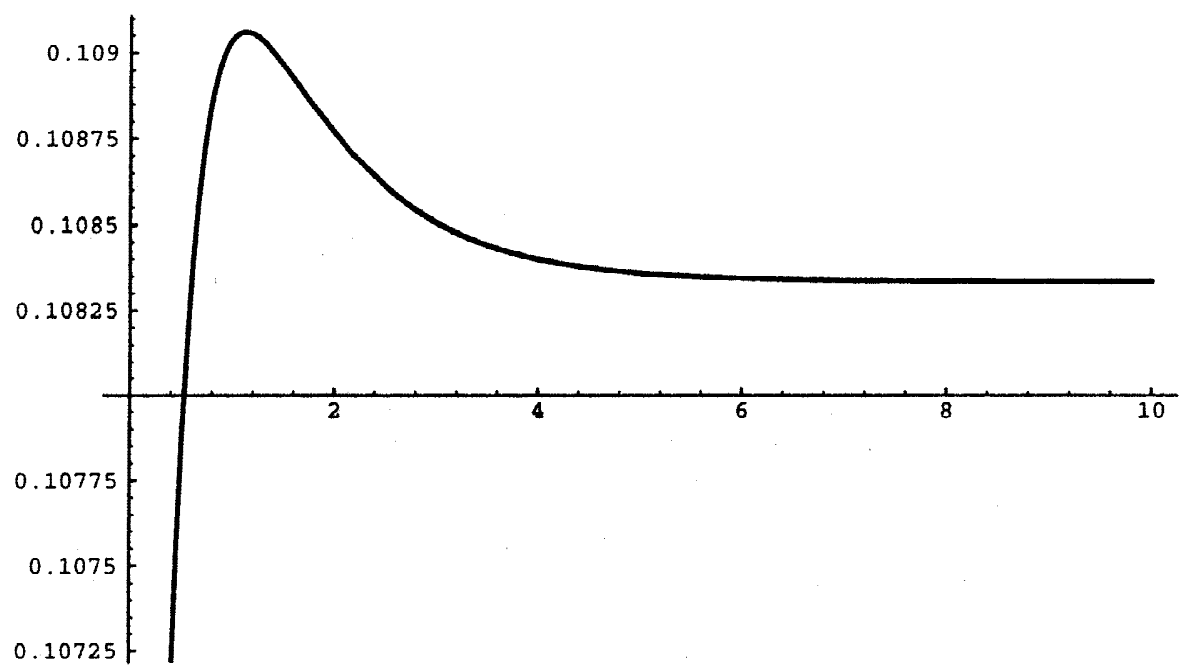

FiguRE 1. Graph of $h(y)$. 
details of such functions. The following characterization of Schur-convex functions will be used to prove Theorem 3.2.

Theorem 3.1. (cf. Marshall and Olkin [12, p. 57]: Let $I \subset R$ be an open interval and let $\phi: I^{n} \rightarrow R$ be continuously differentiable. Necessary and sufficient conditions for $\phi$ to be Schur convex on $I^{n}$ are $\phi$ is symmetric on $I^{n}$ and, for all $i \neq j$,

$$
\left(z_{i}-z_{j}\right)\left[\phi_{(i)}\left(z_{i}\right)-\phi_{(j)}\left(z_{j}\right)\right] \geq 0 \quad \text { for all } z \in I^{n},
$$

where $\phi_{(i)}(z)$ denotes the partial derivative of $\phi$ with respect to its ith argument.

Theorem 3.2: Let $X_{1}, X_{2}$, and $X_{3}$ be independent exponential random variables having hazard rates $\lambda_{1}, \lambda_{2}$, and $\lambda_{3}$, respectively. Then, $\operatorname{cov}\left(D_{2: 3}, D_{3: 3}\right)$ is Schur convex in $\lambda_{i}$ 's.

Proof: The covariance between $D_{2: 3}$ and $D_{3: 3}$ is

$$
\begin{aligned}
\phi\left(\lambda_{1}, \lambda_{2}, \lambda_{3}\right)= & \operatorname{cov}\left(D_{2: 3}, D_{3: 3}\right) \\
= & \left(\lambda_{1} \lambda_{2} \lambda_{3}\right)\left(\lambda_{1}+\lambda_{2}+\lambda_{3}\right)^{-1} \\
& \times\left[\left(\lambda_{1}^{-2}+\lambda_{2}^{-2}\right)\left(\lambda_{1}+\lambda_{2}\right)^{-2}+\left(\lambda_{1}^{-2}+\lambda_{3}^{-2}\right)\left(\lambda_{1}+\lambda_{3}\right)^{-2}\right. \\
& \left.\quad+\left(\lambda_{2}^{-2}+\lambda_{3}^{-2}\right)\left(\lambda_{2}+\lambda_{3}\right)^{-2}\right] \\
- & \left\{( \lambda _ { 1 } + \lambda _ { 2 } + \lambda _ { 3 } ) ^ { - 1 } \left(\lambda_{3} /\left(\lambda_{1}+\lambda_{2}\right)+\lambda_{2} /\left(\lambda_{1}+\lambda_{3}\right)\right.\right. \\
& \left.\left.+\lambda_{1} /\left(\lambda_{2}+\lambda_{3}\right)\right)\right\} \\
\times & \left\{\left(\lambda_{1} \lambda_{2} \lambda_{3}\right)\left(\lambda_{1}+\lambda_{2}+\lambda_{3}\right)^{-1}\right. \\
& \times\left\{\left(\lambda_{2}^{-2}+\lambda_{3}^{-2}\right)\left(\lambda_{2}+\lambda_{3}\right)^{-1}\right. \\
\quad & +\left(\lambda_{1}^{-2}+\lambda_{3}^{-2}\right)\left(\lambda_{1}+\lambda_{3}\right)^{-1} \\
\quad & \left.\left.\quad\left(\lambda_{1}^{-2}+\lambda_{2}^{-2}\right)\left(\lambda_{1}+\lambda_{2}\right)^{-1}\right\}\right\} .
\end{aligned}
$$

After some simplifications, we find that $\left(\lambda_{1}-\lambda_{2}\right)\left\{\phi_{(1)}\left(\lambda_{1}, \lambda_{2}, \lambda_{3}\right)-\right.$ $\left.\phi_{(2)}\left(\lambda_{1}, \lambda_{2}, \lambda_{3}\right)\right\}$ is equal to

$$
\frac{8\left(\lambda_{1}-\lambda_{2}\right)^{2} \lambda_{3}^{2}}{\left(\lambda_{1}+\lambda_{2}\right)\left(\lambda_{1}+\lambda_{3}\right)\left(\lambda_{2}+\lambda_{3}\right)\left(\lambda_{1}+\lambda_{2}+\lambda_{3}\right)},
$$

which is nonnegative for all $\lambda_{1}, \lambda_{2}, \lambda_{3}>0$. Because the function $\phi$ is symmetric in $\left(\lambda_{1}, \lambda_{2}, \lambda_{3}\right)$, the required result follows from Theorem 3.1.

Corollary 3.1: Under the assumptions of Theorem 3.2, $\operatorname{cov}\left(D_{2: 3}, D_{3: 3}\right) \geq 0$ and $\operatorname{var}\left(X_{1: 3}\right) \leq \operatorname{var}\left(X_{2: 3}\right) \leq \operatorname{var}\left(X_{3: 3}\right)$.

Proof: Let $\bar{\lambda}$ be the average of $\lambda_{i}$ 's. It is easy to see that $(\bar{\lambda}, \bar{\lambda}, \bar{\lambda}) \stackrel{m}{\lessgtr}\left(\lambda_{1}, \lambda_{2}, \lambda_{3}\right)$. From Theorem 3.2, we get

$$
\phi(\bar{\lambda}, \bar{\lambda}, \bar{\lambda}) \leq \phi\left(\lambda_{1}, \lambda_{2}, \lambda_{3}\right)
$$


where the function $\phi$ is given by (3.3). The left-hand side of (3.4) is zero, since spacings of a random sample from an exponential distribution are independent. This proves that $\operatorname{cov}\left(D_{2: 3}, D_{3: 3}\right) \geq 0$. Since $D_{1: 3}$ is independent of $D_{2: 3}$ and $D_{3: 3}$, it follows that $\operatorname{cov}\left(X_{3: 3}-X_{2: 3}, X_{2: 3}\right) \geq 0$. The required result follows from Lemma 2.1.

Gross, Hunt, and Odeh [4] considered the single-outlier model in which all except one of the $\lambda_{i}$ 's are equal; that is, $\lambda_{1}=\lambda$ and $\lambda_{2}=\ldots=\lambda_{n}=\lambda^{*}, \lambda \neq \lambda^{*}$. They incorrectly pointed out that in this case, the spacings $D_{i: n}$ and $D_{j: n}$ are independent for $j-i \geq 2$. Although it is true that $D_{1: n}$ is independent of $\left(D_{2: n}, \ldots, D_{n: n}\right)$, the other $D_{i}$ 's are not independent. In fact, for $n=4$,

$$
\operatorname{cov}\left(D_{2: 4}, D_{4: 4}\right)=\frac{2 \lambda^{*}\left(\lambda^{*}-\lambda\right)^{2}}{\left(\lambda^{*}+\lambda\right)\left(2 \lambda^{*}+\lambda\right)^{2}\left(3 \lambda^{*}+\lambda\right)^{2}},
$$

which is positive unless $\lambda^{*}=\lambda$. Theorem 3.4, which follows, replaces the incorrect result of Gross et al. [4] for the single-outlier model.

To prove the remaining results of this section, we shall repeatedly use the following known result.

THEOREM 3.3 (Shaked and Spizzinchino [13]): Let the joint distribution function of $\mathbf{X}=\left(X_{1}, \ldots, X_{n}\right)$ be

$$
F\left(x_{1}, \ldots, x_{n}\right)=\int_{-\infty}^{+\infty} \prod_{i=1}^{n} F_{i}\left(x_{i} \mid \theta_{i}\right) d G(\theta),
$$

where $F_{i}(\cdot \mid \theta)$ is an absolutely continuous distribution function with respect to Lebesgue measure on $R$ for each $\theta$ in the support of $\Theta$ with density function $f_{i}(\cdot \mid \theta)$ for $i=1, \ldots, n$. Suppose that the support of $\left(X_{1}, \ldots, X_{n}\right)$ is a lattice. If $f_{i}(x \mid \theta)$ is $\mathrm{TP}_{2}$ $\left(\mathrm{RR}_{2}\right)$ in $(x, \theta)$ for all $i \in\{1, \ldots, n\}$, then $\left(X_{1}, \ldots, X_{n}\right)$ is $\mathrm{MTP}_{2}$.

In the next theorem, we prove that in the case of a single-outlier exponential model, the spacings are $\mathrm{MTP}_{2}$ dependent.

Theorem 3.4: Let $X_{i}, i=1, \ldots, n$, be independent exponential random variables such that $X_{1}$ has hazard rate $\lambda$ and $X_{i}$ has hazard rate $\lambda^{*}$ for $i \in\{2, \ldots, n\}$. Then, $\left(D_{1: n}, \ldots, D_{n: n}\right)$ is $\mathrm{MTP}_{2}$ dependent.

Proof: Using (3.1), we find that the joint p.d.f. of $\left(D_{1: n}, \ldots, D_{n: n}\right)$ in this case is

$$
\begin{aligned}
f_{D_{1: n}, \ldots, D_{n: n}}\left(x_{1}, \ldots, x_{n}\right)= & \sum_{\theta=1}^{n} \frac{(n-1) ! \lambda\left(\lambda^{*}\right)^{n-1}}{\prod_{i=1}^{\theta}\left((n-i) \lambda^{*}+\lambda\right) \prod_{i=\theta+1}^{n}(n-i+1) \lambda^{*}} \\
& \times \prod_{i=1}^{\theta}\left((n-i) \lambda^{*}+\lambda\right) e^{-\left((n-i) \lambda^{*}+\lambda\right) x_{i}} \\
& \times \prod_{i=\theta+1}^{n}(n-i+1) \lambda^{*} e^{-(n-i+1) \lambda^{*} x_{i}}
\end{aligned}
$$


which can be expressed as

$$
f_{D_{1: n}, \ldots, D_{n: n}}\left(x_{1}, \ldots, x_{n}\right)=\int_{-\infty}^{+\infty} \prod_{i=1}^{n} f_{D_{i: n}}\left(x_{i} \mid \theta\right) d P_{\Theta}(\theta),
$$

where $\Theta$ is a discrete random variable with the probability mass function,

$$
p_{\Theta}(\theta)=\frac{(n-1) ! \lambda\left(\lambda^{*}\right)^{n-1}}{\prod_{i=1}^{\theta}\left((n-i) \lambda^{*}+\lambda\right) \prod_{i=\theta+1}^{n}(n-i+1) \lambda^{*}} \text { for } \theta=1, \ldots, n,
$$

and

$$
f_{D_{i: n}}(x \mid \theta)= \begin{cases}\left((n-i) \lambda^{*}+\lambda\right) e^{-\left((n-i) \lambda^{*}+\lambda\right) x}, & i \leq \theta \\ (n-i+1) \lambda^{*} e^{-(n-i+1) \lambda^{*} x}, & i \geq \theta+1 .\end{cases}
$$

We show that the conditional densities as given by (3.5) are all $\mathrm{TP}_{2}$ if $\lambda<\lambda^{*}$ and are all $\mathrm{RR}_{2}$ if $\lambda>\lambda^{*}$. Suppose $\theta_{1}<\theta_{2}$ and $\theta_{1}, \theta_{2} \in\{1, \ldots, n\}$. Then, the ratio

$$
\frac{f_{D_{i: n}}\left(x \mid \theta_{2}\right)}{f_{D_{i: n}}\left(x \mid \theta_{1}\right)}= \begin{cases}1, & i \leq \theta_{1} \\ \frac{\left((n-i) \lambda^{*}+\lambda\right) e^{-\left((n-i) \lambda^{*}+\lambda\right) x}}{(n-i+1) \lambda^{*} e^{-(n-i+1) \lambda^{*} x}}, & \theta_{1}<i \leq \theta_{2} \\ 1, & \theta_{2}<i\end{cases}
$$

is increasing (decreasing) in $x$ if $\lambda<(>) \lambda^{*}$ for $i=1, \ldots, n$; that is, $f_{D_{i: n}}(x \mid \theta)$ is $\mathrm{TP}_{2}$ $\left(\mathrm{RR}_{2}\right)$ in $(x, \theta)$. The required result follows from Theorem 3.1.

COROLlary 3.2: Under the assumptions of Theorem 3.4,

$$
\operatorname{var}\left(X_{1: n}\right) \leq \operatorname{var}\left(X_{2: n}\right) \leq \cdots \leq \operatorname{var}\left(X_{n: n}\right) .
$$

In the next theorem, we consider the multiple-outliers model. According to this model, $X_{1}, \ldots, X_{k}$ are i.i.d. exponentials with hazard rate $\lambda$ and $X_{k+1}, \ldots, X_{n}$ are i.i.d. exponentials with hazard rate $\lambda^{*}$, where $k \in\{2, \ldots, n-2\}$. We prove that in this case, $D_{i: n}$ and $D_{i+1: n}$ are $\mathrm{TP}_{2}$ dependent for $i=1, \ldots, n-1$. It is not known whether the spacings are $\mathrm{MTP}_{2}$ dependent in this case.

THeORem 3.5: Let $X_{i}, i=1, \ldots, n$, be independent exponential random variables such that $X_{i}$ has hazard rate $\lambda$ for $i \in\{1, \ldots, k\}$ and hazard rate $\lambda^{*}$ for $i \in\{k+1, \ldots, n\}$, $k \in\{2, \ldots, n-2\}$. Then, $D_{i: n}$ and $D_{i+1: n}$ are $\mathrm{TP}_{2}$ dependent.

Proof: Without loss of generality, we assume that $k \leq n-k$.

Case (i): Let $k<i \leq n-k$. From (3.2), the joint p.d.f. of $\left(D_{i: n}, D_{i+1: n}\right)$ for this set of $\lambda_{i}$ 's can be expressed as

$$
f_{D_{i: n}, D_{i+1: n}}(x, y)=\int_{-\infty}^{+\infty} f_{D_{i: n}}(x \mid \theta) f_{D_{i+1: n}}(y \mid \theta) d P_{\Theta}(\theta),
$$


where $\Theta$ is a discrete random variable taking values $0,1,2, \ldots, 2 k$ with the following probability mass function. For $\theta=0,2,4, \ldots, 2 k$,

$$
p_{\Theta}(\theta)=\lambda^{k}\left(\lambda^{*}\right)^{n-k} k !(n-k) ! \sum_{\left(\mathbf{r}_{\theta}\right)} \frac{1}{\prod_{i=1}^{n} \sum_{j=i}^{n} \lambda\left(r_{j}\right)},
$$

where the summation is taken over all permutations of

$$
\left(\mathbf{r}_{\theta}\right)=(\overbrace{\lambda, \ldots, \lambda, \lambda^{*}, \ldots, \lambda^{*}, \lambda^{*}}^{k-\theta / 2} \overbrace{\lambda, \ldots, \lambda}^{i-1-k+\theta / 2} \overbrace{\lambda^{*}, \ldots, \lambda^{*}}^{1})
$$

for which the $i$ th component of $\left(\mathbf{r}_{\theta}\right)$ is $\lambda^{*}$ and its last $n-i$ components consist of $(\theta / 2) \lambda^{\prime}$ s and $(n-i-\theta / 2) \lambda^{*}$ 's.

For $\theta=1,3,5, \ldots, 2 k-1$,

$$
p_{\Theta}(\theta)=\lambda^{k}\left(\lambda^{*}\right)^{n-k} k !(n-k) ! \sum_{\left(\mathbf{r}_{\theta}^{\prime}\right)} \frac{1}{\prod_{i=1}^{n} \sum_{j=i}^{n} \lambda\left(r_{j}^{\prime}\right)},
$$

where the summation is taken over all permutations of

$$
\left(\mathbf{r}_{\theta}^{\prime}\right)=(\overbrace{\lambda, \ldots, \lambda}^{k-(\theta+1) / 2}, \overbrace{\lambda^{*}, \ldots, \lambda^{*}}^{i-1-k+(\theta+1) / 2} \overbrace{\lambda, \lambda, \ldots, \lambda}^{1}, \overbrace{\lambda^{*}, \ldots, \lambda^{*}}^{(\theta+1) / 2-1})
$$

for which the $i$ th component of $\left(\mathbf{r}_{\theta}^{\prime}\right)$ is $\lambda$ and the last $n-i$ components of $\left(\mathbf{r}_{\theta}^{\prime}\right)$ consist of $((\theta+1) / 2-1) \lambda$ 's and $(n-i-(\theta+1) / 2+1) \lambda^{*}$ 's.

For $\theta \in\{0, \ldots, 2 k\}$,

$$
\begin{gathered}
f_{D_{i: n}}(x \mid \theta)=\left\{(n-i-[(\theta+1) / 2]+1) \lambda^{*}+[(\theta+1) / 2] \lambda\right\} \\
\times e^{-\left\{(n-i-[(\theta+1) / 2]+1) \lambda^{*}+[(\theta+1) / 2] \lambda\right\} x}
\end{gathered}
$$

and

$$
f_{D_{i+1: n}}(x \mid \theta)=\left\{(n-i-[\theta / 2]) \lambda^{*}+[\theta / 2] \lambda\right\} e^{-\left\{(n-i-[\theta / 2]) \lambda^{*}+[\theta / 2] \lambda\right\} x},
$$

where $[x]$ denotes the greatest integer less than or equal to $x$.

To prove the required result, we show that $f_{D_{i: n}}(x \mid \theta)$ and $f_{D_{i+1: n}}(x \mid \theta)$ are all $\mathrm{TP}_{2}$ if $\lambda<\lambda^{*}$ and are all $\mathrm{RR}_{2}$ if $\lambda>\lambda^{*}$.

$$
\begin{aligned}
\frac{f_{D_{i: n}}(x \mid \theta+1)}{f_{D_{i: n}}(x \mid \theta)}= & \frac{\left\{(n-i-[(\theta+2) / 2]+1) \lambda^{*}+[(\theta+2) / 2] \lambda\right\} e^{-\left\{(n-i-[(\theta+2) / 2]+1) \lambda^{*}+[(\theta+2) / 2] \lambda\right\} x}}{\left\{(n-i-[(\theta+1) / 2]+1) \lambda^{*}+[(\theta+1) / 2] \lambda\right\} e^{-\left\{(n-i-[(\theta+1) / 2]+1) \lambda^{*}+[(\theta+1) / 2] \lambda\right\} x}} \\
& =\left\{\begin{array}{lc}
1, & \text { if } \theta=1,3,5, \ldots, 2 k-1 \\
\frac{\left\{(n-i-\theta / 2) \lambda^{*}+(\theta / 2+1) \lambda\right\}}{\left\{(n-i-\theta / 2+1) \lambda^{*}+(\theta / 2) \lambda\right\}} e^{-\left(\lambda-\lambda^{*}\right) x}, & \text { if } \theta=0,2,4, \ldots, 2 k-2 .
\end{array}\right.
\end{aligned}
$$


From (3.10), we conclude that if $\lambda<\lambda^{*}\left(\lambda>\lambda^{*}\right)$, then $f_{D_{i: n}}(x \mid \theta)$ is $\operatorname{TP}_{2}\left(\operatorname{RR}_{2}\right)$ for $i=1, \ldots, n$. Similarly, for $f_{D_{i+1: n}}(x \mid \theta)$, we have

$$
\begin{aligned}
& \frac{f_{D_{i+1: n}}(x \mid \theta+1)}{f_{D_{i+1: n}}(x \mid \theta)}=\frac{\left\{(n-i-[(\theta+1) / 2]) \lambda^{*}+[(\theta+1) / 2] \lambda\right\} e^{-\left\{(n-i-[(\theta+1) / 2]) \lambda^{*}+[(\theta+1) / 2] \lambda\right\} x}}{\left\{(n-i-[\theta / 2]) \lambda^{*}+[\theta / 2] \lambda\right\} e^{-\left\{(n-i-[\theta / 2]) \lambda^{*}+[\theta / 2] \lambda\right\} x}} \\
& = \begin{cases}1, & \text { if } \theta=0,2,4, \ldots, 2 k-2 \\
\frac{\left\{(n-i-(\theta+1) / 2) \lambda^{*}+((\theta+1) / 2) \lambda\right\}}{\left\{(n-i-(\theta-1) / 2) \lambda^{*}+((\theta-1) / 2) \lambda\right\}} e^{-\left(\lambda-\lambda^{*}\right) x}, & \text { if } \theta=1,3,5, \ldots, 2 k-1 .\end{cases}
\end{aligned}
$$

Again, from (3.11), it follows that $f_{D_{i+1: n}}(x \mid \theta)$ is $\mathrm{TP}_{2}\left(\mathrm{RR}_{2}\right)$ if $\lambda<\lambda^{*}\left(\lambda>\lambda^{*}\right)$. Using these observations, the required result follows from Theorem 3.1.

Case (ii): $i>n-k$. In this case for $\theta \in\{0,2, \ldots, 2(n-i)\},\left(\mathbf{r}_{\theta}\right)$ is given by (3.6), and for $\theta \in\{1,3, \ldots, 2(n-i)+1\},\left(\mathbf{r}_{\theta}^{\prime}\right)$ is given by (3.7). Hence, for $\theta \in$ $\{0,1,2, \ldots, 2(n-i+1)-1\}, f_{D_{i: n}}(x \mid \theta)$ and $f_{D_{i+1: n}}(x \mid \theta)$ are the same as given by (3.8) and (3.9), respectively. The required result follows from the same kind of arguments as in case (i).

Case (iii): $i \leq k$. The proof is similar to the previous case. The vectors $\left(\mathbf{r}_{\theta}\right)$ and $\left(\mathbf{r}_{\theta}^{\prime}\right)$ corresponding to (3.6) and (3.7) are as follows. For $\theta=0,2, \ldots, 2 i-2$,

$$
\left(\mathbf{r}_{\theta}\right)=(\overbrace{\lambda, \ldots, \lambda, \lambda^{*}, \ldots, \lambda^{*},{ }_{\lambda}^{i-1-\theta / 2}}^{\theta / 2} \overbrace{\lambda, \ldots, \lambda, \lambda^{*}, \ldots, \lambda^{*}}^{1}),
$$

for which the $i$ th component of $\left(\mathbf{r}_{\theta}\right)$ is $\lambda$ and the last $n-i$ components of $\left(\mathbf{r}_{\theta}\right)$ consist of $(k-i+\theta / 2) \lambda$ 's and $(n-k-\theta / 2) \lambda^{*}$ 's.

For $\theta=1,3, \ldots, 2 i-1$,

$$
\left(\mathbf{r}_{\theta}^{\prime}\right)=(\overbrace{\lambda, \ldots, \lambda}^{i-((\theta+1) / 2)}, \overbrace{\lambda^{*}, \ldots, \lambda^{*}}^{((\theta+1) / 2)-1} \overbrace{\lambda^{*}}^{1}, \overbrace{\lambda, \ldots, \lambda}^{k-i+((\theta+1) / 2)}, \overbrace{\lambda^{*}, \ldots, \lambda^{*}}^{n-k-((\theta+1) / 2)}),
$$

for which the $i$ th component of $\left(\mathbf{r}_{\theta}^{\prime}\right)$ is $\lambda^{*}$ and the last $n-i$ components of $\left(\mathbf{r}_{\theta}^{\prime}\right)$ consist of $(k-i+(\theta+1) / 2) \lambda$ 's and $(n-k-(\theta+1) / 2) \lambda^{*}$ 's.

Therefore, for $\theta \in\{0, \ldots, 2 i-1\}$,

$$
\begin{aligned}
f_{D_{i: n}}(x \mid \theta)= & \left\{(k-i+1+[\theta / 2]) \lambda+(n-k-[\theta / 2]) \lambda^{*}\right\} \\
& \times e^{-\left\{(k-i+1+[\theta / 2]) \lambda+(n-k-[\theta / 2]) \lambda^{*}\right\} x}
\end{aligned}
$$

and

$$
\begin{aligned}
f_{D_{i+1: n}}(x \mid \theta)= & \left\{(k-i-[(\theta+1) / 2]) \lambda+(n-k-[(\theta+1) / 2]) \lambda^{*}\right\} \\
& \times e^{-\left\{(k-i-[(\theta+1) / 2]) \lambda+(n-k-[(\theta+1) / 2]) \lambda^{*}\right\} x} .
\end{aligned}
$$

The required result follows from the same kind of arguments as in case (i). 


\section{CONCLUDING REMARKS}

In this paper, we have obtained some new results on dependence among spacings of heterogeneous independent exponential random variables. Whereas in the case of a single-outlier exponential model, the spacings are shown to be $\mathrm{MTP}_{2}$ dependent, it is not known whether the same result holds for the multiple-outliers model. In the latter case, we are only able to establish $\mathrm{TP}_{2}$ dependence between consecutive spacings. Another unsettled question is to examine whether in the case of independent exponential random variables, in general, the spacings are positively correlated. We have given a proof of this conjecture for $n=3$.

\section{Acknowledgments}

One of the authors (B.-E.K.) thanks the Indian Council for Cultural Relations, New Delhi, India, Razi University, Kermanshah, Iran and the Ministry of Culture and Higher Education of the Islamic Republic of Iran, Tehran, Iran for arranging scholarships which enabled him to study at the Indian Statistical Institute, New Delhi, India.

The authors are grateful to the referee for helpful comments and suggestions which greatly improved the presentation of the results.

\section{References}

1. Barlow, R.E. \& Proschan, F. (1981). Statistical theory of reliability and life testing. Silver Spring, MD: To Begin With.

2. Block, H.W. \& Ting, M.L. (1981). Some concepts of multivariate dependence. Communications in Statistics: Theory and Methods 10: 749-762.

3. Esary, J.D. \& Proschan, F. (1972). Relationships among some notions of bivariate dependence. Annals of Mathematical Statistics 43: 651-655.

4. Gross, A.J., Hunt, H.H., \& Odeh, R.E. (1986). The correlation coefficient between the smallest and largest observations when $(n-1)$ of the $n$ observations are i.i.d. exponentially distributed. Сотmunications in Statistics: Theory and Methods 15: 1113-1123.

5. Karlin, S. (1968). Total positivity. Palo Alto, CA: Stanford University Press.

6. Karlin, S. \& Rinott, Y. (1980). Classes of orderings of measures and related correlation inequalities. I. Multivariate totally positive distributions. Journal of Multivariate Analysis 10: 467-498.

7. Karlin, S. \& Rinott, Y. (1980). Classes of orderings of measures and related correlation inequalities. II. Multivariate reverse rule distributions. Journal of Multivariate Analysis 10: 499-516.

8. Kemperman, J.H.B. (1977). On the FKG inequality for measures on a partially ordered space. Indagationes Mathematicae 39: 313-331.

9. Kochar, S.C. (1998). Stochastic comparisons of spacings and order statistics. In A.P. Basu, S.K. Basu, \& S. Mukhopadhyay (eds.), Frontiers in reliability. Singapore: World Scientific, pp. 201-216.

10. Kochar, S.C. \& Korwar, R. (1996). Stochastic orders for spacings of heterogeneous exponential random variables. Journal of Multivariate Analysis 57: 69-83.

11. Lehmann, E.L. (1966). Some concepts of dependence. Annals of Mathematical Statistics 37: 1137-1153.

12. Marshall, A.W. \& Olkin, I. (1979). Inequalities: Theory of majorization and its applications. New York: Academic Press.

13. Shaked, M. \& Spizzinchino, F. (1998). Positive dependence properties of conditionally independent random life times. Mathematics of Operations Research 23: 944-959. 